\title{
Frequency-dependent drag from quantum turbulence produced by quartz tuning forks in superfluid ${ }^{4} \mathrm{He}$
}

\author{
S. L. Ahlstrom, D. I. Bradley, M. Človečko, S. N. Fisher, A. M. Guénault, E. A. Guise, R. P. Haley, O. Kolosov, \\ P. V. E. McClintock, G. R. Pickett, M. Poole, V. Tsepelin, ${ }^{*}$ and A. J. Woods \\ Department of Physics, Lancaster University, Lancaster, LAI 4YB, United Kingdom
}

(Received 12 November 2013; revised manuscript received 23 December 2013; published 30 January 2014)

\begin{abstract}
We have measured the drag force from quantum turbulence on a series of quartz tuning forks in superfluid helium. The tuning forks were custom made from a $75-\mu \mathrm{m}$-thick wafer. They have identical prong widths and prong spacings, but different lengths to give different resonant frequencies. We have used both the fundamental and overtone flexure modes to probe the turbulent drag over a broad range of frequencies $f=\omega / 2 \pi$ from 6.5 to $300 \mathrm{kHz}$. Optical measurements show that the velocity profiles of the flexure modes are well described by a cantilever beam model. The critical velocity for the onset of quantum turbulence at low temperatures is measured to be $v_{c} \approx \sqrt{0.7 \kappa \omega}$ where $\kappa$ is the circulation quantum. The drag from quantum turbulence shows a small frequency dependence when plotted against the scaled velocity $v / v_{c}$.
\end{abstract}

DOI: 10.1103/PhysRevB.89.014515

PACS number(s): 67.25.dk, 67.25.dt, 47.27.Cn, 47.37.+q

\section{INTRODUCTION}

Quantum turbulence, a tangle of quantized vortex lines in a superfluid, has received a great deal of attention in recent years [1-3]. There is mounting evidence from experiments [4-9] and computer simulations [10-12] that quantum turbulence shares many of the essential properties of classical turbulence. At very low temperatures, in the absence of any normal fluid component, quantum turbulence is particularly simple: it consists of an irregular network of quantum vortices in which each vortex carries the same circulation and moves according to the sum of its own flow field, the flow fields from all neighboring vortices, and any externally applied flow. There is no viscosity at low temperatures, but quantum turbulence can still dissipate by creating excitations. Excitations may be created by vortex reconnections and by the resulting high-frequency Kelvin waves. The mechanisms depend on the nature of the superfluid: sound (phonon) emission occurs in superfluid ${ }^{4} \mathrm{He}$ [13] and quasiparticle emission in superfluid ${ }^{3} \mathrm{He}[14,15]$.

Quantum turbulence at low temperatures can be generated by a mechanical oscillator. In superfluid ${ }^{4} \mathrm{He}$, the drag force on a mechanical oscillator at high velocities is approximately proportional to the square of the velocity corresponding to a constant drag coefficient $C_{D}$, as is normally found for turbulent drag in classical fluids. In a classical fluid, oscillating objects do not normally have a clearly defined critical velocity for the onset of turbulence: on increasing velocity, the flow becomes progressively more complex as it transforms from laminar flow at low velocities to turbulence at high velocities. There is often a broad crossover region with many strikingly different flow regimes, while the drag coefficient varies smoothly with velocity $[16,17]$.

At low velocities, the dissipative drag force in laminar flow arises from viscosity and is commonly known as Stokes drag.

\footnotetext{
*v.tsepelin@lancaster.ac.uk

Published by the American Physical Society under the terms of the Creative Commons Attribution 3.0 License. Further distribution of this work must maintain attribution to the author(s) and the published article's title, journal citation, and DOI.
}

The Stokes drag on an object oscillating at a high frequency $f=\omega / 2 \pi$ is given by

$$
C_{D}^{L}=c_{L} \frac{\sqrt{\omega \nu}}{v},
$$

where $v$ is the kinematic viscosity and $c_{L}$ is a geometrical constant of order unity. This formula is valid when the viscous penetration depth $\delta=\sqrt{2 v / \omega}$ is small compared to the dimensions of the object, which is easily fulfilled for typical tuning forks in normal liquid helium. In classical fluids, the turbulent drag at high velocities $C_{D}^{T}$ is approximately equal to a constant of order unity $\simeq c_{T}$. Equating the turbulent drag with the Stokes drag gives a crossover velocity

$$
v_{\times} \simeq \frac{c_{L}}{c_{T}} \sqrt{\omega \nu}
$$

In contrast, a superfluid at low temperatures displays pure potential flow at low velocities with no viscous drag. In this case, the drag is dominated by intrinsic losses which may be measured in vacuum [18]. Furthermore, in superfluid ${ }^{4} \mathrm{He}$ the onset of "turbulent" drag usually occurs at a well-defined critical velocity $v_{c}$. Here, we use the term turbulent drag loosely to indicate drag arising from the creation and/or growth of vortex lines. It is not necessary for the flow to be strictly turbulent in the sense of having irregular motion of a vortex tangle. For instance, drag may be produced by the emission of vortex rings, as is known to occur for a grid oscillating in superfluid ${ }^{3} \mathrm{He}-\mathrm{B}[19]$.

There is mounting evidence, from decay measurements $[5,6,20,21]$ that quantum turbulence behaves similarly to turbulence in a classical fluid with an effective kinematic viscosity, the magnitude of which is normally written as a multiple of the circulation quantum $\kappa=h / m$ where $m$ is the mass of a ${ }^{4} \mathrm{He}$ atom for superfluid ${ }^{4} \mathrm{He}$, or twice the mass of a ${ }^{3} \mathrm{He}$ atom for superfluid ${ }^{3} \mathrm{He}$. Further similarities between quantum and classical turbulence are revealed by theoretical arguments [13,22-24], by experiments which probe fluctuations in the flow [4,7,9], and by computer simulations [12,25].

By analogy with Eq. (2) for classical turbulence, one might anticipate a critical velocity for the onset of quantum 
turbulence at low temperatures of the form

$$
v_{c}=\sqrt{\beta \omega \kappa},
$$

where $\beta$ is a constant of order unity, dependent on the flow geometry.

The frequency dependence of the critical velocity given in Eq. (3) can be derived using dynamical scaling arguments for vortex dynamics. These arguments were first used to explain the critical velocity for turbulence production by high-amplitude second sound waves [26,27]. Other arguments can also be used to derive a similar result [28]. The first experimental evidence for a critical velocity given by Eq. (3) was obtained with oscillating spheres [29,30]. We note, however, that Eq. (3) should only be applicable at relatively high frequencies where the amplitude of oscillation $a=v / \omega$ is small compared to the size of the object $R$. This is applicable in most cases, including the measurements presented in the following. At lower frequencies, where the amplitude of oscillation exceeds the dimensions of the object $R$, we would expect the critical velocity to become independent of frequency [31] $v_{c} \approx \kappa / R$. This was found to be the case for a low-frequency oscillating grid in superfluid ${ }^{4} \mathrm{He}$ [32].

Quartz tuning forks have been widely used for turbulence studies in helium superfluids [33-36]. Most of the work has been performed on forks with resonant frequencies around $32 \mathrm{kHz}$ and with various geometries and measurement conditions resulting in a range of critical velocities. Here, we present a detailed study of the critical velocity and the turbulent drag in superfluid ${ }^{4} \mathrm{He}$ at very low temperatures using a carefully designed set of tuning forks. The forks were custom manufactured to give a broad range of resonant frequencies with nominally identical geometries, differing only in the length of the prongs. We have also studied turbulent behavior at different frequencies for the same fork by measuring different resonance modes.

\section{EXPERIMENTAL SETUP}

The experimental cell is shown schematically in Fig. 1(a). The inner cell body is made from a cylindrical rod of Araldite epoxy resin. This is potted in a larger cylinder of Stycast 1266 (not shown in the figure) to strengthen the cell for experiments at high pressures. The experimental volume, with a width of $15 \mathrm{~mm}$ and a height of approximately $21 \mathrm{~mm}$, was machined to have a square cross section with rounded corners as indicated. It was filled with isotopically pure liquid ${ }^{4} \mathrm{He}$ [37]. The cell was cooled by a silver sinter heat exchanger connected to a much larger heat exchanger in the mixing chamber of the dilution refrigerator via a $\sim 20$-cm-long 1-mm-diameter annealed silver wire. The assembly of custom-made quartz tuning forks [38] was mounted vertically along one side of the experimental volume, as indicated in the figure. The cell also contains several other devices which were not used for the current experiments, but are shown for completeness: a large lithium niobate tuning fork, a floppy wire resonator, and two pressure sensors. Experiments using these devices are described elsewhere [39,40].

Figure 1(b) shows a picture of the tuning fork assembly. The array of five forks labeled A1-A5 share a common pair of leads. The four single quartz tuning forks labeled S1-S4 have
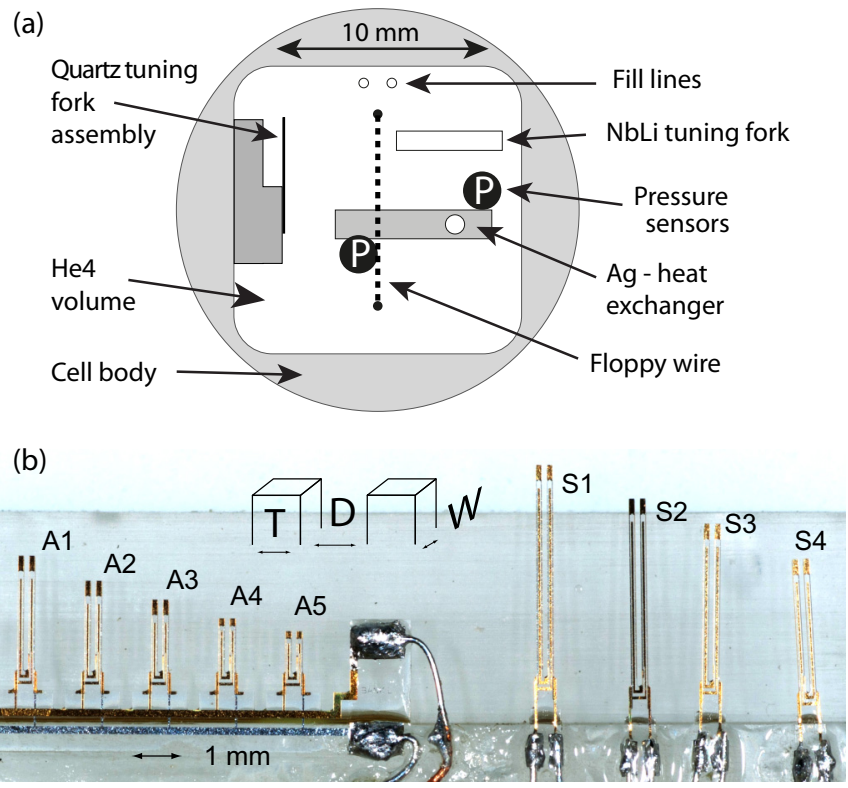

FIG. 1. (Color online) (a) Sketch of the experimental cell. The tuning forks are mounted along the vertical length of the left-hand wall of the cell. (b) Image of the tuning fork assembly; forks A1-A5 are on a single array with common leads; S1-S4 are single tuning forks with separate leads. See text.

separate leads. The forks are aligned in a row as shown and glued to an Araldite plastic support using Stycast 1266 epoxy. Earlier measurements found that the intrinsic damping of the forks can be increased significantly by flexing of the support. To avoid this, the support was made very rigid. The tuning forks have prongs with a common width $W=75 \mu \mathrm{m}$, determined by the thickness of quartz wafer used to manufacture them. Their prongs also have a common thickness of $T=90 \mu \mathrm{m}$ and the spacing between the prongs is $D=90 \mu \mathrm{m}$. The prong length is varied from $L=0.7$ to $3.5 \mathrm{~mm}$ to give different fundamental resonant frequencies for each fork spanning a wide range, approximately 6 to $160 \mathrm{kHz}$. Electrical connections were made by soldering $75-\mu \mathrm{m}$-diameter copper wire to the contact pads.

A schematic of the measurement circuit is given in Fig. 2. The tuning fork operation relies on the piezoelectric properties of the quartz. The driving force on the prongs is supplied by an applied voltage and the resulting velocity generates a current. Each tuning fork was driven by a function generator. The output voltage of the generator was attenuated by up to $60 \mathrm{~dB}$ to allow very small driving forces. The current generated by the fork motion was measured using a custom-made currentto-voltage converter [41] and a lock-in amplifier.

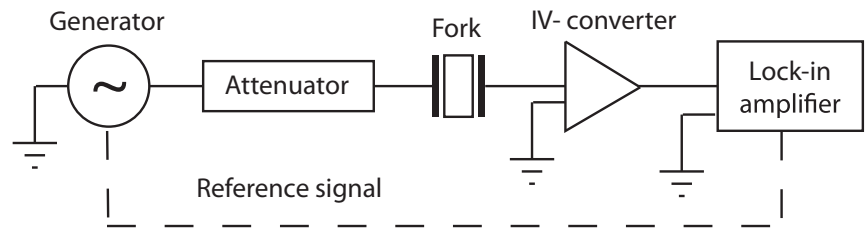

FIG. 2. Schematic of the measurement circuit. 


\section{MODELING AND CALIBRATING THE PRONG VELOCITY}

The quartz tuning fork prong velocity can be estimated from the signal current using the measured electrical properties as described in the following. The velocity can also be measured directly using optical techniques. Previous measurements of the prong tip velocity of tuning forks in the fundamental resonance mode yielded approximately $10 \%$ agreement between optical and electrical techniques [36,42]. Following, we present optical measurements of the velocity profile along the length of the fork prongs for various resonant modes and compare the tip velocity with that inferred from electrical measurements. First, we give the predictions of a simple cantilever model for the prong motion and describe how the tip velocity is estimated from the electrical properties.

\section{A. Cantilever beam model}

We can model each prong as a thin dissipationless oscillating beam of length $L$, thickness $T$, and width $W$. The beam is rigidly clamped at one end. The equation of motion with a harmonic drive uniformly distributed along the beam can be written as [43]

$$
\mu \frac{\partial^{2} u(x, t)}{\partial t^{2}}+\frac{\partial^{2}}{\partial x^{2}}\left(E I \frac{\partial^{2} u(x, t)}{\partial x^{2}}\right)=q_{0} e^{i \omega t},
$$

where $u(x, t)$ is the displacement of the beam at position $x$ along its length, $\mu$ is the mass per unit length, $E=7.87 \times$ $10^{10} \mathrm{~N} / \mathrm{m}^{2}$ is the Young's modulus of quartz, $I$ is the area moment of inertia, and $q_{0}$ is the driving force per unit length. For a beam of uniform rectangular cross section, $I=W T^{3} / 12$ and $\mu=\rho_{q} W T$ where $\rho_{q}=2659 \mathrm{kgm}^{-3}$ is the density of quartz.

Solutions of Eq. (4) with the appropriate boundary conditions can be written in the form $u(x, t)=X^{n}(x) e^{i \omega_{n} t}$. The resonant frequencies are given by [43]

$$
\omega_{n}=2 \pi f_{n}=\sqrt{\frac{E I}{\mu}} b_{n}^{2}=\sqrt{\frac{E T^{2}}{12 \rho}} b_{n}^{2},
$$

where the wave number $b_{n}$ of each flexural resonant mode is determined by solutions to

$$
\cos \left(b_{n} L\right) \cosh \left(b_{n} L\right)+1=0 .
$$

Numerical solutions for the fundamental and the first three overtone modes give $b_{0} L=1.875, b_{1} L=4.694, b_{2} L=$ 7.855, and $b_{3} L=10.996$, respectively.

The displacement profile along the beam for a given resonance mode $n$ can be written as $X^{n}(x)=X_{r}^{n}(x) X^{n}(L)$ where the fractional displacement relative to the prong tip is given by [43]

$$
\begin{aligned}
X_{r}^{n}(x)= & A\left[\cosh \left(b_{n} x\right)-\cos \left(b_{n} x\right)\right] \\
& -B\left[\sinh \left(b_{n} x\right)-\sin \left(b_{n} x\right)\right],
\end{aligned}
$$

where

$$
\begin{aligned}
A & =\frac{1}{2} \frac{\sin \left(b_{n} L\right) \sinh \left(b_{n} L\right)}{\cosh \left(b_{n} L\right)+\cos \left(b_{n} L\right)}, \\
B & =\frac{1}{2} \frac{\cos \left(b_{n} L\right) \sinh \left(b_{n} L\right)+\sin \left(b_{n} L\right) \cosh \left(b_{n} L\right)}{\cosh \left(b_{n} L\right)+\cos \left(b_{n} L\right)} .
\end{aligned}
$$

\section{B. Electromechanical model}

To describe the resonant properties of a tuning fork in a fluid, it is useful to model each prong as a damped harmonic oscillator with an effective mass $m_{\text {eff }}$ and spring constant $k$. The equation of motion is written as

$$
\frac{d^{2} u^{n}(t)}{d t^{2}}+\gamma \frac{d u^{n}(t)}{d t}+\omega_{n}^{2} u^{n}(t)=\frac{F(t)}{m_{\mathrm{eff}}},
$$

where $u^{n}(t)$ is the displacement of the tip of each prong, $\gamma$ describes the drag force, $F(t)=F_{0} e^{i \omega t}$ is the driving force, and $\omega_{n}^{2}=k_{n} / m_{\text {eff }}$ is the resonant frequency in vacuum. Modeling each prong as an ideal cantilever, the effective mass is independent of the flexural mode and equal to one quarter of its actual mass [43]

$$
m_{\mathrm{eff}}=\frac{\mu L}{4}=\frac{\rho_{q} L W T}{4} .
$$

In a fluid, $\gamma$ has both real and imaginary components $\gamma=\gamma_{2}+i \gamma_{1}$. The real component $\gamma_{2}$ describes the dissipative drag forces while the imaginary component $\gamma_{1}$ describes the nondissipative force arising from the backflow fluid around the prongs. The latter may be considered to act as an additional effective mass which shifts the resonant frequency.

Solving Eq. (8) for the tip velocity $v^{n}=d u^{n} / d t=v_{0}^{n} e^{i \omega t}$ yields a velocity amplitude

$$
v_{0}^{n}=\frac{F_{0}}{m_{\mathrm{eff}}} \frac{\gamma_{2} \omega^{2}-i \omega\left(\omega_{n}^{2}-\omega^{2}-\omega \gamma_{1}\right)}{\left(\omega_{n}^{2}-\omega^{2}-\omega \gamma_{1}\right)^{2}+\gamma_{2}^{2} \omega^{2}} .
$$

Under typical conditions, and at low velocities, the fluid drag force is relatively small and is proportional to the prong velocity. In this case, $\gamma_{1}$ and $\gamma_{2}$ are constants much smaller than the resonant frequency, and Eq. (10) then describes a Lorentzian resonance line of width $\Delta f_{2}=\gamma_{2} /(2 \pi)$. The maximum tip velocity amplitude occurs at resonance and is given by

$$
v_{r}=\frac{F_{0}}{m_{\mathrm{eff}} \gamma_{2}} \simeq \frac{F_{0}}{2 \pi m_{\mathrm{eff}} \Delta f_{2}} .
$$

The driving force on each prong can be written as $F=$ $a V / 2$, where $a$ is the fork constant [44]. The prong motion stresses the quartz which results in a piezoelectric current $I$. On resonance, the driving force is balanced by the dissipative drag force on the two prongs, so the power dissipated is given by $\dot{Q}=2 F v=I V$. Consequently, the current is given by $I=$ $a v$. Comparing with the harmonic oscillator model [Eq. (11)], the fork constant is found to be

$$
a=\sqrt{\frac{4 \pi m_{\mathrm{eff}} \Delta f_{2} I_{r}}{V_{r}}},
$$

where $I_{r}$ and $V_{r}$ are the current and voltage amplitudes at the resonant frequency, respectively. The fork constant can thus be determined from the measured resonance line and the fork dimensions assuming that the effective mass is given by Eq. (9).

In practice, measurements of the current utilize a phasesensitive lock-in referenced to the drive generator as shown in Fig. 2. The in-phase and quadrature components of the current correspond to the real and imaginary components of the velocity given in Eq. (10). 


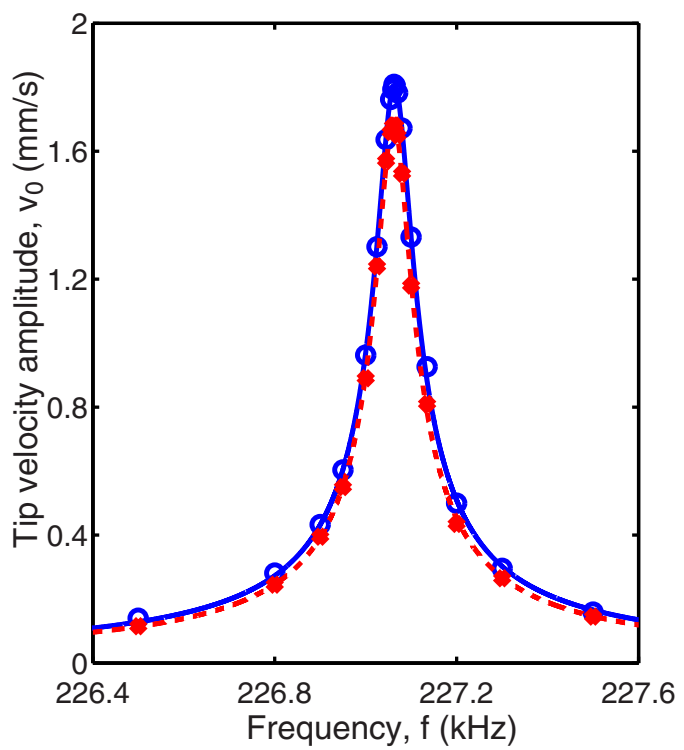

FIG. 3. (Color online) Comparison of electromechanical (open blue circles) and optical (closed red circles) measurements of the tip velocity versus frequency for the third harmonic of an S1-type quartz tuning fork. Lines give fits to the ideal line shape [Eq. (10)].

\section{Measurements of the tip velocity}

Optical measurements were made at room temperature on S1-type $(L=3.5 \mathrm{~mm})$ and $\mathrm{S} 4$-type $(L=2.2 \mathrm{~mm})$ tuning forks. A laser Doppler shift vibrometer was used to measure the velocity profile of the prong at the location of an incident laser. The laser was focused to a spot diameter of around $20 \mu \mathrm{m}$ which determined the spatial resolution.

Figure 3 shows measurements of the tip velocity amplitude versus frequency for the third harmonic of an S1-type tuning fork. The figure also shows the velocity inferred from simultaneous electrical measurements of the fork constant using Eq. (12), where the frequency width $\Delta f_{2}$ and the resonant current $I_{r}$ are obtained from fits to the in-phase and quadrature line shapes of the form given by Eq. (10). The two techniques agree to within a few percent. The small differences may be accounted for by uncertainties in the effective mass $m_{\text {eff }}$ arising from the fork geometry (in practice, the prongs are slightly irregular).

A summary of the optical and electromechanical measurements is given in Table I. The first column labels the fork type and the flexure mode. The second column gives the resonant frequency. The third and fourth columns show the fitted resonant width for electromechanical and optical methods, respectively. The fork constant calculated using Eq. (12) is listed in the fifth column. The last three columns show the electromechanical and optical resonant velocity amplitudes and their ratio, respectively. In most cases, the electromechanical calibration slightly overestimates the velocity, but the agreement is usually better than $10 \%$.

\section{Velocity profiles}

To measure the profile of the velocity amplitude along the length of the fork prong, we use a micromanipulator to adjust and measure the position of the fork relative to the focused
TABLE I. Comparison of electromechanical and optical measurements of the prong tip velocity for S1 and S4 tuning forks (see text).

\begin{tabular}{lccccccc}
\hline \hline Mode & $\begin{array}{c}f_{0} \\
(\mathrm{~Hz})\end{array}$ & $\begin{array}{c}\Delta f_{2}^{\mathrm{el}} \\
(\mathrm{Hz})\end{array}$ & $\begin{array}{c}\Delta f_{2}^{\mathrm{opt}} \\
(\mathrm{Hz})\end{array}$ & $\begin{array}{c}a \times 10^{7} \\
\left(\mathrm{Cm}^{-1}\right)\end{array}$ & $\begin{array}{c}v_{\mathrm{el}} \\
\left(\mathrm{mm} \mathrm{s}^{-1}\right)\end{array}$ & $\begin{array}{c}v_{\text {opt }} \\
\left(\mathrm{mm} \mathrm{s}^{-1}\right)\end{array}$ & $v_{\mathrm{el}} / v_{\text {opt }}$ \\
\hline S1F0 & 6708.4 & 7.7 & 8.2 & 3.80 & 2.39 & 2.24 & 1.07 \\
S1F1 & 41932 & 14.9 & 14.9 & 14.7 & 4.76 & 4.85 & 0.98 \\
S1F2 & 116801 & 24.1 & 23.3 & 18.2 & 3.65 & 3.66 & 1.00 \\
S1F3 & 227063 & 80.5 & 77.7 & 30.1 & 1.81 & 1.69 & 1.07 \\
S4F0 & 16934 & 10.4 & 10.7 & 5.65 & 4.15 & 3.89 & 1.04 \\
S4F1 & 105460 & 22.9 & 22.3 & 22.1 & 7.43 & 7.12 & 1.04 \\
S4F2 & 291568 & 42.5 & 44.0 & 19.5 & 3.54 & 2.89 & 1.23 \\
\hline \hline
\end{tabular}

laser spot. Figure 4 shows the measured velocity profiles for an S1-type fork with $L=3.5 \mathrm{~mm}$. Measurements are shown for the fundamental mode and the first three harmonics.

According to the cantilever beam approximation, the velocity profile should be given by $v^{n}(x)=X_{r}^{n}(x) v^{n}(L)$ where $X_{r}^{n}(x)$ is given by Eq. (7). The solid lines in Fig. 4 show the expected velocity profile $v^{n}(x)$ according to this expression, where the tip velocity $v^{n}(L)$ is the only fitting parameter. The cantilever models show excellent agreement with the measured profiles.

\section{LOW-TEMPERATURE CHARACTERIZATION}

The tuning forks were characterized in superfluid ${ }^{4} \mathrm{He}$ at temperature $T=450 \mathrm{mK}$ and 22-bar pressure. The temperature was chosen since it corresponds to the zero-temperature limit for studying the drag from quantum turbulence, as shown below, while the mechanical time constants of the resonant modes are no more than a few seconds. At the lowest temperatures, the time constants of the low-frequency modes become much longer so that accurate measurements take a very long time and are more difficult.

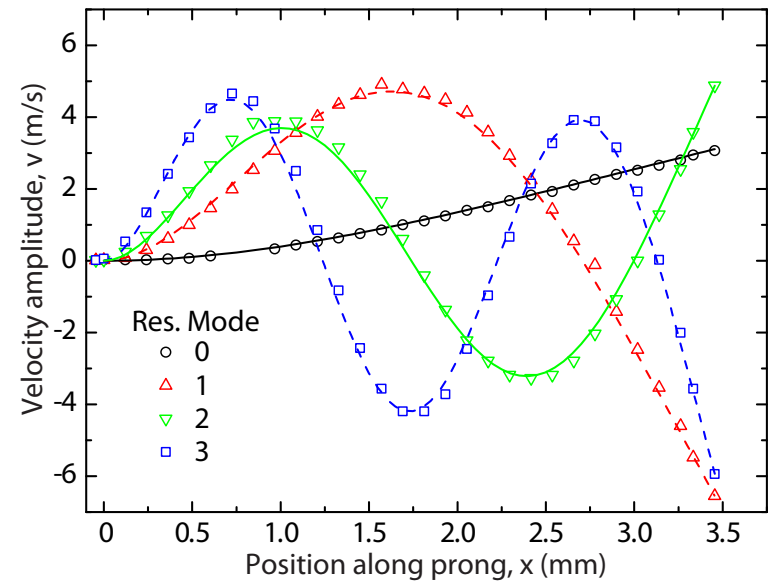

FIG. 4. (Color online) The velocity amplitude of an S1-type tuning fork versus position along the length of one of its prongs. The measurements were made at room temperature with a laser Doppler shift vibrometer. Results are shown for the first four resonance modes. The lines show the displacement profile predicted by Eq. (7). 
TABLE II. Properties of the fork resonance modes at low velocities in superfluid helium at $450 \mathrm{mK}$. The first column lists the fork, the second gives the prong length, and the third column gives the resonant mode, where " 0 " corresponds to the fundamental, " 1 ," " 2 ," and " 3 " correspond to the first, second, and third overtone modes, respectively. The fourth column gives the resonant frequency. The fifth column gives the width of the resonance and the final column gives the fork constant determined by Eq. (12).

\begin{tabular}{lccccc}
\hline \hline $\begin{array}{c}L \\
\text { Fork }\end{array}$ & $\begin{array}{c}L \\
(\mu \mathrm{m})\end{array}$ & Mode & $\begin{array}{c}f_{0} \\
(\mathrm{kHz})\end{array}$ & $\begin{array}{c}\Delta f_{2} \\
(\mathrm{~Hz})\end{array}$ & $\begin{array}{c}a \times 10^{7} \\
\left(\mathrm{Cm}^{-1}\right)\end{array}$ \\
\hline S1 & 3500 & 0 & 6.488 & 0.1 & 3.35 \\
& & 1 & 40.492 & 0.3 & 15.8 \\
& & 2 & 112.818 & 1.1 & 18.3 \\
S2 & 3100 & 0 & 219.268 & 30 & 28.8 \\
& & 1 & 5.267 & 0.1 & 4.16 \\
& & 2 & 143.547 & 0.2 & 18.8 \\
& & 3 & 278.440 & 58 & 19.8 \\
S4 & 2200 & 0 & 16.439 & 0.2 & 5.98 \\
& & 1 & 102.279 & 1.1 & 23.9 \\
& & 2 & 282.148 & 101 & 21.8 \\
A1 & 1900 & 0 & 21.946 & 0.2 & 6.53 \\
& & 1 & 135.873 & 4.6 & 24.6 \\
A3 & 1200 & 0 & 54.166 & 0.2 & 9.31 \\
A4 & 900 & 0 & 95.354 & 1.4 & 10.2 \\
A5 & 700 & 0 & 155.448 & 2.8 & 13.7 \\
\hline \hline
\end{tabular}

The tuning fork resonances were characterized by slowly sweeping the frequency through the resonance at a low driving force. The resonance line shapes were fitted to the expected Lorentzian response given by Eq. (10). The fits were excellent in all cases except for fork A2 which had a double-peak resonance and was therefore excluded from further studies. The results are summarized in Table II.

The measured resonant frequencies of all the forks and all the different flexure modes agree to within $4 \%$ of the calculated frequencies using Eq. (5). The small discrepancies can be accounted for by the presence of the electrodes and by uncertainties in the prong geometries. The fork constants shown in the table are very similar to values obtained in vacuum at $4.2 \mathrm{~K}$ and in air at room temperature.

The frequency width of each resonance is proportional to the dissipative drag force per unit velocity. In superfluid ${ }^{4} \mathrm{He}$ at $450 \mathrm{mK}$ the drag at low velocities and low frequencies is dominated by intrinsic losses and by scattering with ballistic phonons as discussed in the following. At higher frequencies, above $100 \mathrm{kHz}$, the resonance widths increase rapidly due to additional drag produced by the emission of acoustic phonons. The acoustic drag is consistent with earlier studies and is well described by theoretical models of acoustic emission in an infinite fluid $[43,45]$. However, since the wavelength of the acoustic emission is typically smaller than the experimental cell, it is possible to excite standing waves. Acoustic standing waves may have many closely spaced resonances depending on the cell geometry and these may couple to the tuning fork resonances resulting in complex behavior [45-47]. The effects are very strong when the fork resonance overlaps with the frequency of acoustic modes and is most readily observed as a splitting of the resonance into multiple peaks. Measurements to compare the behavior of different forks were performed at 22 bar where the effects of the coupling were found to be largely absent, although there may have been some residual effects at higher velocities as discussed in the following.

\section{TURBULENT DRAG}

The set of quartz tuning forks allows us to study superfluid flow over a very broad range of frequencies, for nearly identical geometries apart from the prong length $L$. Here, our main focus is on the drag from quantum turbulence.

Figure 5 shows the tip velocity amplitude of fork $\mathrm{S} 1$, at the fundamental resonance, versus the driving force for various temperatures in superfluid ${ }^{4} \mathrm{He}$ at 0 -bar pressure. In practice, the resonant frequency varies a little with the velocity. At resonance, the driving force is exactly balanced by the dissipative drag force and the quadrature signal is zero according to Eq. (10). So, to maintain resonance the frequency is adjusted at each measurement point to minimize the quadrature signal.

At low velocities, the velocity is proportional to the drag force. The turbulent drag increases more rapidly with velocity above some onset velocity. The onset is quite gradual at high temperatures but becomes sharp at low temperatures. Furthermore, at low temperatures the transition to turbulence often exhibits hysteresis. On increasing the driving force, the velocity drops abruptly when turbulent drag is nucleated. On reducing the drive, the velocity decreases towards the values measured in the potential flow state, but there remains a very small upward step in velocity corresponding to an abrupt transition back to the pure potential flow state. Similar behavior has been observed for vibrating wires [48,49] and vibrating spheres [50]. In the following, we use "down" sweeps taken with reducing drive so we can measure the turbulent drag to lower velocities.

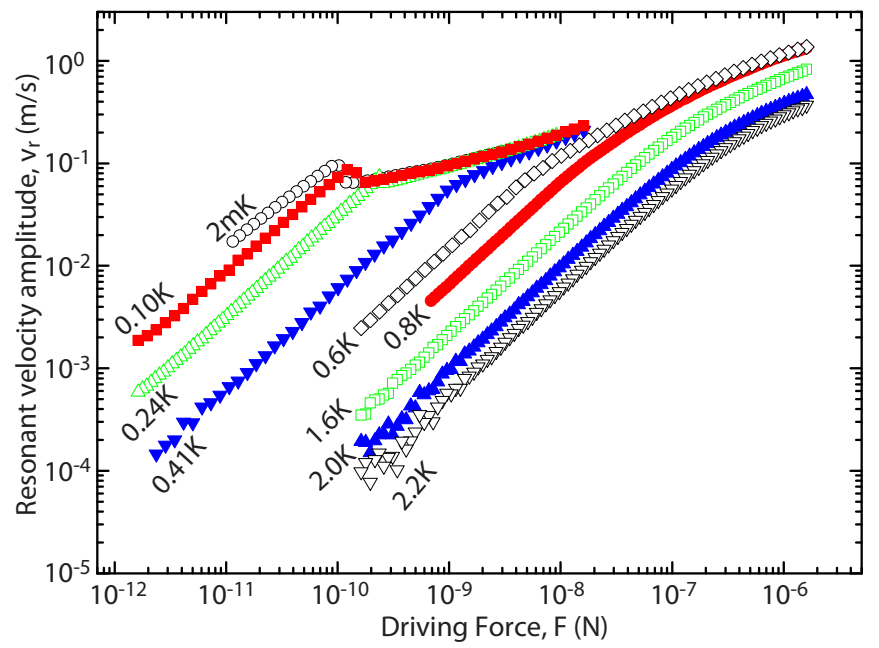

FIG. 5. (Color online) The resonant tip velocity amplitude of the fundamental mode of fork S1 versus the driving force for various temperatures at 0 -bar pressure. The data at $2.2 \mathrm{~K}$ are in normal liquid ${ }^{4} \mathrm{He}$. 


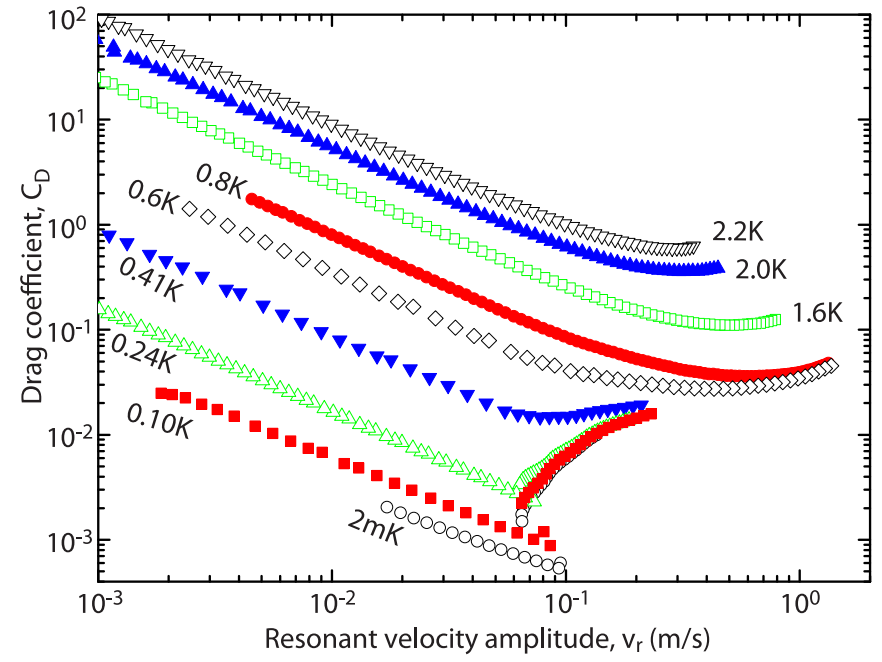

FIG. 6. (Color online) The drag coefficient, inferred from the data in Fig. 5, as function of velocity for various temperatures.

We define the drag coefficient as

$$
C_{D}=\frac{2 F_{0}}{\rho_{\mathrm{He}} W L v_{r}^{2}},
$$

where $F_{0}$ is the amplitude of the driving force at resonance. We note that when the drag force is nonlinear, it can not balance the driving force at all times. For the measurements presented here, the forks always have a high- $Q$ factor so their velocity response will always be sinusoidal despite the nonlinear drag force. At resonance, the mean power dissipated in the fluid must equate to the mean power supplied $I_{r} V_{r} / 2=F_{0} v_{r} / 2$. So, the driving force amplitude can be equated to a suitably averaged mean drag force.

Figure 6 shows the drag coefficient $C_{D}$ for the $\mathrm{S} 1$ fork versus the tip velocity amplitude for various temperatures, corresponding to the data shown in Fig. 5. At low velocities the drag force is proportional to the velocity, so the drag coefficient varies as $C_{D} \propto v^{-1}$. At temperatures above $1 \mathrm{~K}$, the lowvelocity drag is due to laminar flow of the viscous normal fluid component and is well described by Stokes drag [44]. At lower temperatures, the mean-free path of the thermal phonons which make up the normal fluid component becomes larger than the prong width and so the drag becomes dominated by the scattering of ballistic phonons [50]. At the very lowest temperatures, the drag is dominated by intrinsic processes and is comparable to that measured in vacuum at $4 \mathrm{~K}$.

At higher velocities, additional drag appears due to turbulence. The flow of a classical fluid around oscillating cylinders has been studied in detail, revealing a rich phase diagram, but the drag coefficient behaves smoothly and tends towards a constant value of order unity at high velocities [16,51]. The measurements at the highest temperature in Fig. 6, in normal liquid ${ }^{4} \mathrm{He}$, show similar behavior. On decreasing temperature in superfluid ${ }^{4} \mathrm{He}$ the drag coefficient falls over the whole range of velocities as the normal fluid component decreases. This indicates that the drag is largely dominated by the normal fluid component and is diminished by the superfluid component even in the presence of turbulence. Similar behavior was previously reported for a vibrating wire resonator [52], but very different behavior has been observed for other tuning forks [34]. The latter work found that the drag coefficient at high velocities was almost temperature independent. Indeed, one might anticipate that at very high velocities the drag will not depend on whether the fluid is superfluid or normal. Our measurements, however, show that for these forks the superfluid drag is much reduced over a considerable range of velocities where the normal fluid shows turbulent drag. Furthermore, for temperatures slightly below the superfluid transition, the drag is described quite well by the diluted normal fluid component alone, indicating that the superfluid might not be turbulent at all. If the two fluids were very well coupled by mutual friction, we would expect the small superfluid component at high temperatures to follow the classical flow pattern of the normal fluid and there would be little change in the drag coefficient. We thus conclude that the two fluids must be decoupled in this case.

At low temperatures, the thermal (phonon) excitations become highly ballistic. Here, the two-fluid model is not applicable since there is no longer a normal "fluid" component, just a gas of independent ballistic excitations which can not produce any turbulent drag. If we suppose that there is no significant coupling between the turbulent superfluid component and the ballistic phonons, then we can define the drag coefficient for pure superfluid turbulence as

$$
C_{D}^{T}=\frac{2\left(F-\alpha v_{r}\right)}{L W \rho_{\mathrm{He}} v_{r}^{2}},
$$

where $\alpha$ is a constant at a given temperature. The term $\alpha v_{r}$ represents the linear drag force due to the sum of intrinsic losses, phonon emission where applicable at high frequencies, and ballistic phonons. It is obtained by a fit to the data at low velocities. We note that such a procedure can not be applied in the two-fluid regime since the laminar and turbulent contributions to the normal fluid drag are not additive.

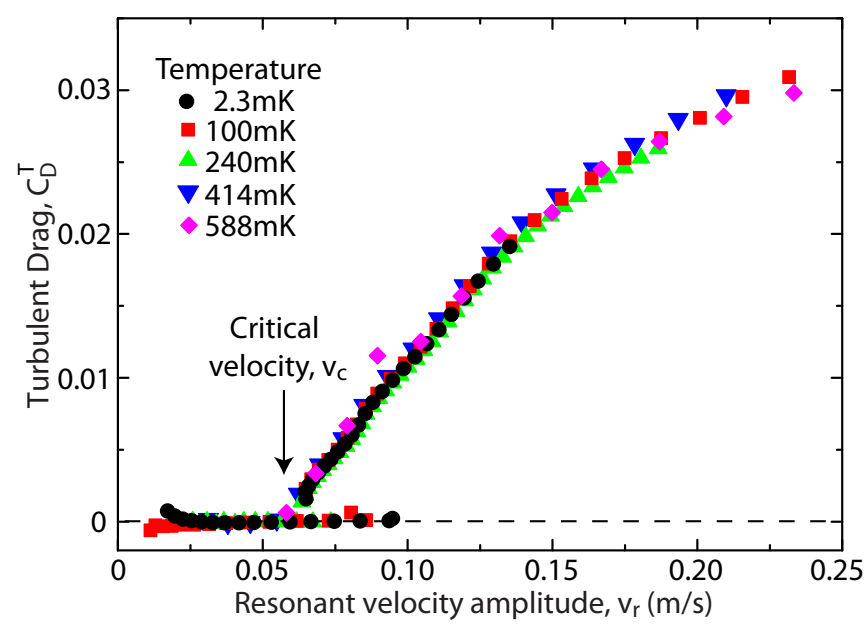

FIG. 7. (Color online) The turbulent drag coefficient, inferred from the data in Fig. 5, versus the prong tip velocity for down sweeps at various low temperatures. Note that the velocity steps upwards at the transition to potential flow where the turbulent drag vanishes. The data collapse onto a single curve showing that the turbulent drag at low temperatures is unaffected by ballistic phonons. 
In Fig. 7, we plot the drag coefficient due to pure superfluid turbulence $C_{D}^{T}$ versus the prong tip velocity at various temperatures below $0.6 \mathrm{~K}$. The data collapse onto a single curve. This confirms that ballistic phonons are largely unaffected by vortex lines. Phonons are known to scatter off vortex cores as this is the mechanism for mutual friction at low temperatures. However, since the cross section is very small [53], only a very small fraction of phonons will be affected by the vortices giving a negligible affect on the ballistic phonon drag. The collapse of the data in Fig. 7 also shows that the turbulent drag from the superfluid is temperature independent in the ballistic regime. Deviations are observed at higher temperatures, for $T \gtrsim 0.8 \mathrm{~K}$, in the two-fluid hydrodynamic regime where the phonon mean-free path becomes shorter than the prong dimensions.

\section{FREQUENCY DEPENDENCE OF THE CRITICAL VELOCITY}

To obtain the critical velocity $v_{c}$, we extrapolate the turbulent drag to zero to find the velocity at which it vanishes, indicated in Fig. 7 for the fundamental resonance of fork S1. This gives a well-defined velocity for a given set of conditions, while the velocities for the appearance and disappearance of the turbulence vary from sweep to sweep, presumably due to different configurations of remanent vortices pinned to surface roughness on the prongs.

Figure 8 shows the velocity-force response for various modes of the S1 fork at a temperature of $400 \mathrm{mK}$. At low frequencies, the critical velocity $v_{c}$ is clear and pronounced. At higher frequencies, however, the onset is less pronounced due to the large acoustic drag. For the third overtone mode, the acoustic drag was so large that we were unable to reach the critical velocity. Furthermore, at high frequencies, anomalous behavior is often observed in the vicinity of the critical velocity, similar to that reported previously $[39,47]$. It has been speculated that the anomalous behavior might be due to nonlinear coupling to acoustic modes [47] and that remanent vortices spanning the prongs might also play a role [39]. Whatever their origin, these effects make it very difficult to identify a clear critical velocity. The measurements presented

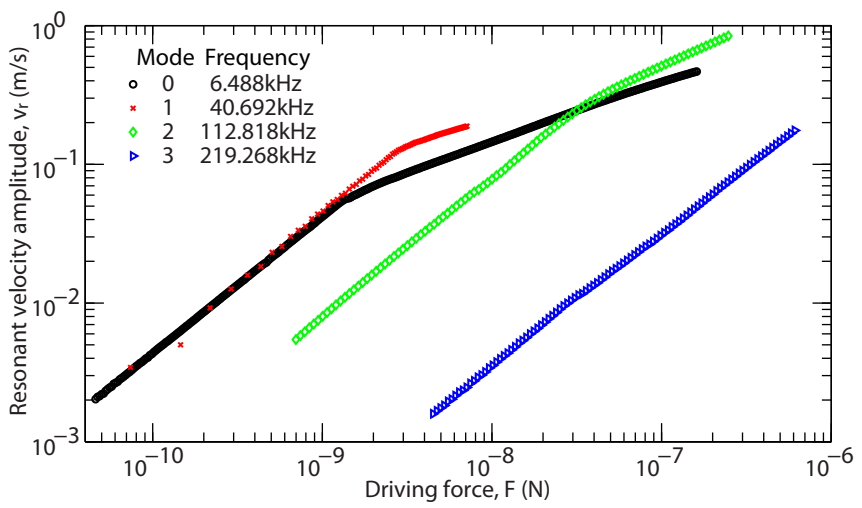

FIG. 8. (Color online) The tip velocity amplitude $v_{r}$ versus the driving force for various resonances of the S1 fork in superfluid ${ }^{4} \mathrm{He}$ at 22 bar pressure and a temperature of $400 \mathrm{mK}$. The drag arising from acoustic emission becomes dominant at the higher frequencies.

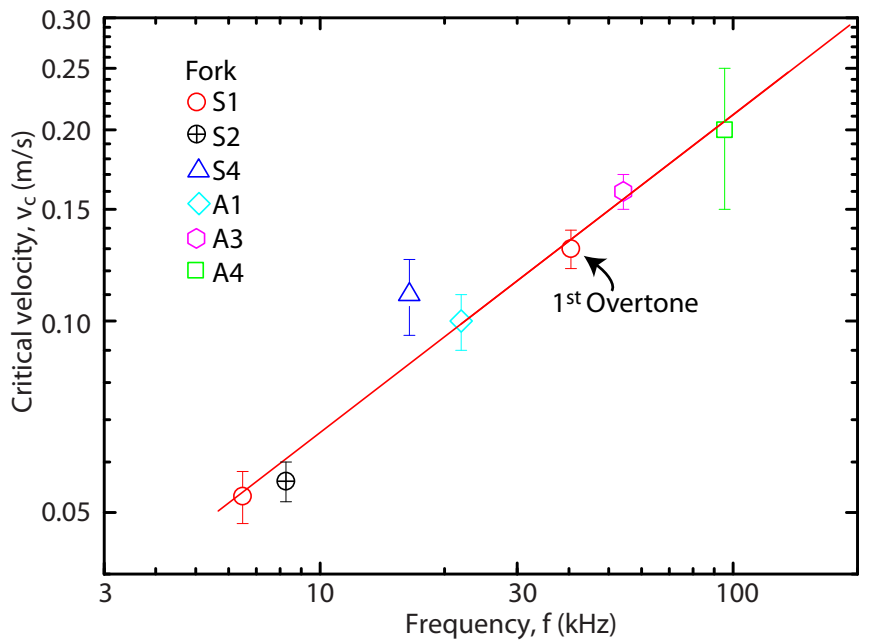

FIG. 9. (Color online) The critical velocity versus the resonant frequency for various forks. The solid line shows $v_{c}=\sqrt{0.73 \kappa \omega}$. The points correspond to the fundamental modes except where indicated.

here were therefore confined to data which show a sharp critical velocity and no anomalous damping.

Figure 9 shows the critical velocity $v_{c}$ versus frequency. The critical velocity is well described by $v_{c} \approx \sqrt{0.73 \kappa \omega}$. The data were mostly taken using the fundamental resonance modes of the forks, but there is a single point taken for the first overtone of the $\mathrm{S} 1$ fork. The good agreement indicates that the critical velocity is governed mainly by the tips of the tuning fork prongs and is insensitive to the velocity profile of the prongs.

\section{FREQUENCY DEPENDENCE OF TURBULENT DRAG}

Figure 10 shows the turbulent drag for the fundamental modes plotted versus the prong tip velocity amplitude scaled by the critical velocity given in Fig. 9. The data span more than a decade in frequency, from 6.5 to $95 \mathrm{kHz}$. The figure reveals a small dependence on frequency; the turbulent drag at the higher reduced velocities tends to be smaller for higher frequencies. The magnitude of the drag is much less than unity over the whole frequency range and is much lower than the drag measured at higher temperatures shown in

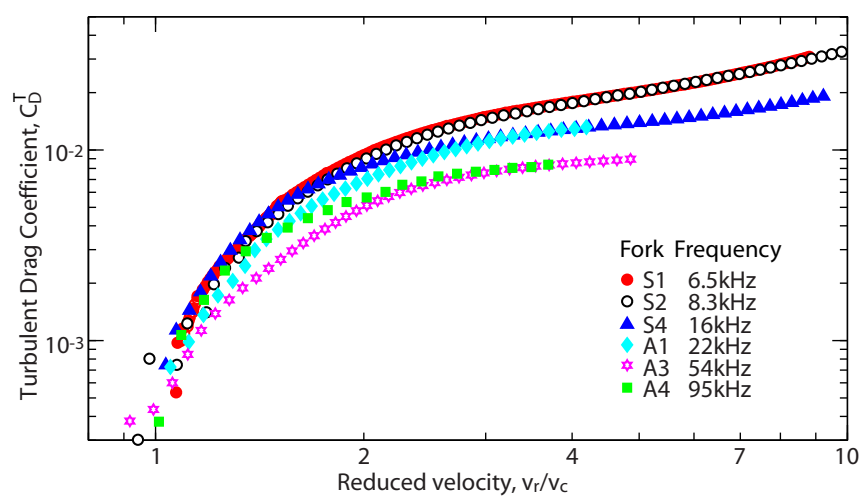

FIG. 10. (Color online) The turbulent drag at low temperatures versus the reduced velocity $v_{r} / v_{c}$ for the fundamental mode of various forks, spanning a large range of frequencies. 
Fig. 6, even for velocities 10 times higher than the critical velocity. This demonstrates very clearly that, over a wide velocity range, the drag from quantum turbulence is much lower than that from classical turbulence. A similarly small drag coefficient has been found for vibrating wire resonators at low temperatures [48].

\section{SUMMARY AND DISCUSSION}

We have presented detailed measurements of custom-made $75-\mu \mathrm{m}$ quartz tuning forks in superfluid helium ${ }^{4} \mathrm{He}$ over a wide temperature range. By varying the length of the tuning fork prongs, we have studied the resonant response over a broad range of frequencies. The frequency range is extended further by studying higher resonant modes of the forks. Optical measurements of the velocity profiles of the fork prongs show that the resonant modes are well described by a simple cantilever model, and the absolute velocities can be determined to within an accuracy of $\sim 10 \%$ from electromechanical measurements. We have investigated the fluid drag on the tuning forks in several distinct regimes. We first summarize the behavior at higher temperatures and at low frequencies.

For temperatures above $1 \mathrm{~K}$, the drag is dominated by the normal fluid component. The drag arises from viscous laminar flow at low velocities and by more complex flow at higher velocities. The drag falls very significantly over the entire velocity range on reducing the temperature in the superfluid phase. So, for these particular tuning forks, the normal and superfluid components must be substantially decoupled and the turbulent drag from the superfluid is relatively small.

At low temperatures, the drag at low velocities is due to scattering with ballistic phonons. Here, there is a well-defined critical velocity $v_{c}$ for the onset of "turbulent" drag from the superfluid component. The turbulent drag must arise from the production of quantum vortices which we expect to become turbulent at sufficiently high velocities. Previous measurements with tuning forks $[35,47]$ and wires [48] identified two critical velocities at low temperatures. The onset of superfluid drag occurred at the first critical velocity $v_{c 1}$ but rose slowly at first. A much faster increase in the drag occurred at the second critical velocity $v_{c 2}$. It was speculated that $v_{c 1}$ marks the onset of drag from stretching and possible reconnections of independent remanent vortices pinned to surface roughness, while $v_{c 2}$ marks the onset of an extended tangle of vortices (quantum turbulence). In the current experiments, we observe a single critical velocity and the superfluid drag remains much smaller than unity even at the highest velocities (in Fig. 10, $C_{D}^{T} \approx 0.03$ at a velocity $v_{r}=10 v_{c}$ ). This may indicate that $v_{c 2}$ is much higher for these forks and the turbulence is not fully developed.

The turbulent drag in the ballistic phonon regime at low temperatures is independent of temperature showing that the superfluid and (ballistic) normal components are almost entirely decoupled. This results from the very small cross section for scattering phonons by a vortex line at low temperatures [53].

At higher frequencies, there is additional drag from acoustic emission. The acoustic drag force is quite linear in velocity and, in most cases, simply adds to the hydrodynamic/ballistic drag. The turbulent drag plotted versus the reduced velocity $v / v_{c}$ shows a weak dependence on the frequency. These measurements were made at 22-bar pressure, but we note that the turbulent drag and the critical velocity are found to be quite insensitive to pressure [35].

The critical velocities for the onset of turbulent drag at low temperatures are well described by $v_{c}=\sqrt{\beta \kappa \omega}$ with $\beta=0.73$. This dependence can be qualitatively explained by scaling laws applied to vortex dynamics [26-28]. A similar dependence was previously reported for oscillating spheres $[29,30]$, but with a much larger prefactor in the range $6.8<\beta<8.1$. The maximum velocity of the fluid backflow around the object depends on geometry. The local fluid velocity is enhanced around sharp corners, thus reducing the critical velocity. So, the lower critical velocity of our tuning forks might be due to the relatively sharp edges of the fork prongs.

The onset of turbulence at low temperatures is known to be controlled by remanent vortices [48,49]. We might therefore expect that the critical velocity will also depend on the available pinning sites for remanent vortices, i.e., the surface roughness. In superfluid ${ }^{4} \mathrm{He}$, the coherence length which governs the size of the vortex core is of atomic dimensions, $\sim 0.1 \mathrm{~nm}$. Most surfaces will be very rough on this length scale, with many available pinning sites to trap remanent vortices. Above the critical velocity, the trapped remanent vortices must become unstable. The vortices will grow and self-reconnect to emit vortex rings. Vortex ring emission has been observed directly in oscillating grid measurements in superfluid ${ }^{3} \mathrm{He}$ [19] and in vibrating wire measurements in superfluid ${ }^{4} \mathrm{He}$ [54]. The subsequent fate of the vortex rings will depend on geometry, on temperature, and on the rate of ring emission. At high temperatures, the rings decay by mutual friction, but this is negligible at low temperatures [55] At low temperatures and low ring emission rates, the rings will propagate away ballistically, either colliding with the cell walls or with another part of the oscillating object, e.g., with the other prong in the case of a tuning fork. In the latter case, the rings will supply vortex line to occupy other pinning cites which may act as seeds for further ring production. At sufficiently high ring emission rates, the rings may collide with other rings. In this case, larger and therefore slower rings are generated which are more likely to suffer further collisions, triggering an avalanche of reconnections and the development of an extended vortex tangle (quantum turbulence) [11,19]. It is likely that fully developed quantum turbulence is necessary to produce a large drag coefficient of order unity. The small values observed in the current experiments at low temperatures may therefore indicate ballistic ring emission without an extended vortex tangle.

Our understanding of turbulence has progressed a great deal in recent years. However, a quantitative understanding of the transition to turbulence for a macroscopic vibrating object poses a formidable task. It is likely to depend on geometry, on surface roughness, and on the availability of remanent vortices. Fortunately, the problem is aided by computer simulations $[11,56]$. Recent simulations have been used to calculate the turbulent drag force on a smooth sphere at low temperatures [57]. In that case, vortices had to be artificially introduced to trigger the turbulence. It would be interesting to extend this work to study the effects of surface roughness and geometry. 


\section{ACKNOWLEDGMENTS}

We thank S. M. Holt, A. Stokes, and M. G. Ward for excellent technical support, and P. Skyba for useful discussions. This research was supported by the European
FP7 Programme MICROKELVIN Project No. 228464, UK EPSRC Grants No. EP/G030596/1, No. EP/L000016/1, No. EP/I028285/1, the NSF/EPSRC Material World Network under Grant No. EP/H04762x/1, the Leverhulme Trust under Grant No. F/00185/X.
[1] Quantized Vortex Dynamics and Superfluid Turbulence, edited by C. F. Barenghi, R. J. Donnelly, and W. Vinen (Springer, Berlin, 2001).

[2] W. F. Vinen and J. J. Niemela, J. Low Temp. Phys. 128, 167 (2002).

[3] S. Fisher and G. Pickett, Prog. Low Temp. Phys. 16, 147 (2009).

[4] J. Maurer and P. Tabeling, Europhys. Lett. 43, 29 (1998).

[5] D. I. Bradley, D. O. Clubb, S. N. Fisher, A. M. Guénault, R. P. Haley, C. J. Matthews, G. R. Pickett, V. Tsepelin, and K. Zaki, Phys. Rev. Lett. 96, 035301 (2006).

[6] P. M. Walmsley, A. I. Golov, H. E. Hall, A. A. Levchenko, and W. F. Vinen, Phys. Rev. Lett. 99, 265302 (2007).

[7] D. I. Bradley, S. N. Fisher, A. M. Guénault, R. P. Haley, S. O'Sullivan, G. R. Pickett, and V. Tsepelin, Phys. Rev. Lett. 101, 065302 (2008).

[8] D. I. Bradley, S. N. Fisher, A. M. Guénault, R. P. Haley, G. R. Pickett, D. Potts, and V. Tsepelin, Nat. Phys. 7, 473 (2011).

[9] P. Roche, P. Diribarne, T. Didelot, O. Français, L. Rousseau, and H. Willaime, Europhys. Lett. 77, 66002 (2007).

[10] M. Tsubota and M. Kobayashi, Prog. Low Temp. Phys. 16, 1 (2009).

[11] S. Fujiyama, A. Mitani, M. Tsubota, D. I. Bradley, S. N. Fisher, A. M. Guénault, R. P. Haley, G. R. Pickett, and V. Tsepelin, Phys. Rev. B 81, 180512 (2010).

[12] A. W. Baggaley, J. Laurie, and C. F. Barenghi, Phys. Rev. Lett. 109, 205304 (2012).

[13] W. F. Vinen, J. Low Temp. Phys. 145, 7 (2006).

[14] M. A. Silaev, Phys. Rev. Lett. 108, 045303 (2012).

[15] M. A. Silaev, J. Low Temp. Phys. 171, 539 (2013).

[16] B. Uzunoğlu, M. Tan, and W. G. Price, Int. J. Numer. Methods Eng. 50, 2317 (2001).

[17] M. Tatsuno and P. W. Bearman, J. Fluid Mech. 211, 157 (1990).

[18] D. I. Bradley, D. O. Clubb, S. N. Fisher, A. M. Guénault, R. P. Haley, C. J. Matthews, G. R. Pickett, and K. L. Zaki, J. Low Temp. Phys. 138, 493 (2005).

[19] D. I. Bradley, D. O. Clubb, S. N. Fisher, A. M. Guénault, R. P. Haley, C. J. Matthews, G. R. Pickett, V. Tsepelin, and K. Zaki, Phys. Rev. Lett. 95, 035302 (2005).

[20] S. R. Stalp, L. Skrbek, and R. J. Donnelly, Phys. Rev. Lett. 82, 4831 (1999).

[21] L. Skrbek, J. J. Niemela, and R. J. Donnelly, Phys. Rev. Lett. 85, 2973 (2000).

[22] V. L'vov, S. Nazarenko, and L. Skrbek, J. Low Temp. Phys. 145, 125 (2006).

[23] E. V. Kozik and B. V. Svistunov, J. Low. Temp. Phys. 156, 215 (2009).

[24] S. K. Nemirovskii, Phys. Rep. 524, 85 (2013).

[25] N. Sasa, T. Kano, M. Machida, V. S. L'vov, O. Rudenko, and M. Tsubota, Phys. Rev. B 84, 054525 (2011).

[26] V. Kotsubo and G. W. Swift, Phys. Rev. Lett. 62, 2604 (1989).

[27] V. Kotsubo and G. Swift, J. Low Temp. Phys. 78, 351 (1990).

[28] R. Hänninen and W. Schoepe, J. Low Temp. Phys. 164, 1 (2011).
[29] R. Hänninen and W. Schoepe, arXiv:0801.2521.

[30] R. Hänninen and W. Schoepe, J. Low Temp. Phys. 153, 189 (2008).

[31] R. Hänninen and W. Schoepe, J. Low Temp. Phys. 158, 410 (2010).

[32] D. I. Bradley, S. N. Fisher, A. M. Guénault, R. P. Haley, M. Kumar, C. R. Lawson, R. Schanen, P. V. E. McClintock, L. Munday, G. R. Pickett et al., Phys. Rev. B 85, 224533 (2012).

[33] G. A. Sheshin, A. A. Zadorozhko, E. Y. Rudavskii, V. K. Chagovets, L. Skrbek, and M. Blazhkova, Low Temp. Phys. 34, 875 (2008).

[34] M. Blažková, D. Schmoranzer, L. Skrbek, and W. F. Vinen, Phys. Rev. B 79, 054522 (2009).

[35] D. I. Bradley, M. J. Fear, S. N. Fisher, A. M. Guénault, R. P. Haley, C. R. Lawson, P. V. E. McClintock, G. R. Pickett, R. Schanen, V. Tsepelin et al., J. Low. Temp. Phys. 156, 116 (2009).

[36] V. Efimov, D. Garg, O. Kolosov, and P. McClintock, J. Low Temp. Phys. 158, 456 (2010).

[37] P. C. Hendry and P. V. E. McClintock, Cryogenics 27, 131 (1987).

[38] Statek Corporation, California.

[39] S. L. Ahlstrom, D. I. Bradley, M. Človečko, S. N. Fisher, A. M. Guénault, E. A. Guise, R. P. Haley, M. Kumar, P. V. E. McClintock, G. R. Pickett, E. Polturak, M. Poole, I. Todoshchenko, V. Tsepelin, and A. J. Woods, J. Low Temp. Phys. (2013), doi:10.1007/s10909-013-0930-6.

[40] S. L. Ahlstrom, D. I. Bradley, M. Človečko, S. N. Fisher, A. M. Guénault, E. A. Guise, R. P. Haley, M. Kumar, P. V. E. McClintock, G. R. Pickett, E. Polturak, M. Poole, I. Todoshchenko, V. Tsepelin, and A. J. Woods, J. Low Temp. Phys. (2013), doi:10.1007/s10909-013-0922-6.

[41] S. Holt and P. Skyba, Rev. Sci. Instrum. 83, 064703 (2012).

[42] D. I. Bradley, P. Crookston, M. J. Fear, S. N. Fisher, G. Foulds, D. Garg, A. M. Guénault, E. Guise, R. P. Haley, O. Kolosov et al., J. Low. Temp. Phys. 161, 536 (2010).

[43] D. I. Bradley, M. Človečko, S. N. Fisher, D. Garg, E. Guise, R. P. Haley, O. Kolosov, G. R. Pickett, V. Tsepelin, D. Schmoranzer et al., Phys. Rev. B 85, 014501 (2012).

[44] R. Blaauwgeers, M. Blazkova, M. Človečko, V. B. Eltsov, R. de Graaf, J. Hosio, M. Krusius, D. Schmoranzer, W. Schoepe, L. Skrbek et al., J. Low Temp. Phys. 146, 537 (2007).

[45] D. Schmoranzer, M. La Mantia, G. Sheshin, I. Gritsenko, A. Zadorozhko, M. Rotter, and L. Skrbek, J. Low Temp. Phys. 163, 317 (2011).

[46] A. Salmela, J. Tuoriniemi, and T. Rysti, J. Low Temp. Phys. 162, 678 (2011).

[47] D. Garg, V. B. Efimov, M. Giltrow, P. V. E. McClintock, L. Skrbek, and W. F. Vinen, Phys. Rev. B 85, 144518 (2012).

[48] D. I. Bradley, S. N. Fisher, A. M. Guénault, R. P. Haley, V. Tsepelin, G. R. Pickett, and K. L. Zaki, J. Low Temp. Phys. 154, 97 (2009). 
[49] H. Yano, T. Ogawa, A. Mori, Y. Miura, Y. Nago, K. Obara, O. Ishikawa, and T. Hata, J. Low Temp. Phys. 156, 132 (2009).

[50] J. Jäger, B. Schuderer, and W. Schoepe, Phys. Rev. Lett. 74, 566 (1995).

[51] T. Sarpkaya, J. Fluid Mech. 165, 61 (1986).

[52] D. I. Bradley, A. M. Guénault, S. N. Fisher, R. P. Haley, M. J. Jackson, D. Nye, K. O'Shea, G. R. Pickett, and V. Tsepelin, J. Low Temp. Phys. 162, 375 (2011).

[53] A. L. Fetter, Phys. Rev. A 136, 1488 (1964).
[54] Y. Nago, A. Nishijima, H. Kubo, T. Ogawa, K. Obara, H. Yano, O. Ishikawa, and T. Hata, Phys. Rev. B 87, 024511 (2013).

[55] D. I. Bradley, S. N. Fisher, A. M. Guénault, R. P. Haley, C. J. Matthews, G. R. Pickett, J. Roberts, S. O'Sullivan, and V. Tsepelin, J. Low Temp. Phys. 148, 235 (2007).

[56] R. Hänninen, M. Tsubota, and W. F. Vinen, Phys. Rev. B 75, 064502 (2007).

[57] S. Fujiyama and M. Tsubota, Phys. Rev. B 79, 094513 (2009). 